\title{
Diarrea persistente en países en vías de desarrollo
}

\author{
Zulfiqar A. Bhutta \\ Departamento de Pediatría y Salud Infantil, Universidad Aga Khan, Karachi, Pakistán
}

\section{Palabras clave}

Diarrea persistente $\cdot$ Deficiencia de micronutrientes .

Desnutrición · Inmunodeficiencia · Tratamiento,

inapropiado, diarrea aguda

\section{Extracto}

Globalmente, las enfermedades diarreicas representan casi $1 / 5$ de todas las muertes de niños menores de 5 años, con una estimación de 2,2 millones de fallecimientos anuales. De estas muertes, una proporción significativa guarda relación con episodios de diarrea persistente (DP; definida como una diarrea que persiste más de 14 días con crecimiento vacilante). Se ha demostrado que la DP identifica a niños con una carga diarreica considerablemente mayor. Estos representan del 36 al 54\% de todas las muertes relacionadas con diarrea. Aunque la patogénesis exacta de la DP sigue sin conocerse a ciencia cierta, la mayoría de los episodios está relacionada con una diarrea infecciosa aguda previa en la que se prolonga el episodio más reciente. Esto puede guardar relación con varios factores del hospedante, como subnutrición preexistente, deficiencia de micronutrientes, inmunodeficiencia y tratamiento inapropiado del episodio diarreico agudo más reciente. El acontecimiento final común parece una lesión prolongada de la mucosa intestinal y una reparación ineficaz. En contraste con la DP postinfecciosa, algunos lactantes pueden presentar diarrea intratable grave debido a diversos trastornos, muchos de los cuales comparten una base hereditaria. Ésta constituye actualmente el tipo dominante de diarrea prolongada en los países desarrollados. El tratamiento de la DP depende de una minuciosa atención a la rehabilitación nutricional y a la identificación precoz de las complicaciones. Aunque se han asociado la DP con diversos patógenos, el papel del tratamiento antimicrobiano por vía oral en la terapéutica sistemática de la DP es exiguo. No obstante, la atención a la rehidratación, la detección y el tratamiento apropiados de las infecciones sistémicas y la rehabilitación nutricional enteral con dietas fácilmente digeribles son elementos cruciales en el tratamiento de estos niños, frecuentemente en entornos ambulatorios. La administración de cinc y vitamina A puede acortar la duración de la DP y contribuir también a reducir las recidivas. No obstante, las estrategias preventivas más importantes para la DP son la lactancia materna exclusiva durante los 6 primeros meses de vida, el mantenimiento de dicha lactancia durante 2 años con nutrición complementaria apropiada y el tratamiento óptimo de los episodios diarreicos agudos.

Copyright (c) 2006 Nestec Ltd., Vevey/S. Karger AG, Basel

\begin{tabular}{ll}
\hline KARGER & @ 2006 Nestec Ltd., Vevey/S. Karger AG, Basel \\
Fax +41 61 306 12 34 & 0252-8185/06/0641-0039\$23.50/0 \\
$\begin{array}{l}\text { E-Mail karger@karger.ch } \\
\text { www.karger.com }\end{array}$ & $\begin{array}{l}\text { Accesible online en: } \\
\text { www.karger.com/asn }\end{array}$
\end{tabular}

Zulfiqar Ahmed Bhutta

Department of Paediatrics and Child Health

Aga Khan University, PO Box 3500, Stadium Road

Karachi 74800 (Pakistan)

Tel. +92 21 4930051/ext. 4724, Fax +92 21 4934294, E-Mail zulfiqar.bhutta@aku.edu 


\section{Introducción}

A pesar de los adelantos considerables realizados en el conocimiento y el tratamiento de los trastornos diarreicos de la infancia, éstos representan todavía una elevada proporción $(20 \%)$ de muertes infantiles, con una estimación de 2,2 millones de fallecimientos [1]. En una estimación global de la carga de trastornos diarreicos en 1980, la Organización Mundial de la Salud calculó que tenían lugar más de 700 millones de episodios de diarrea al año en niños menores de 5 años en países en vías de desarrollo (excluyendo China), con una cifra aproximada de 4,6 millones de muertes [2]. Las revisiones más recientes de los estudios publicados en los últimos 10 años indican que, a pesar de la posible reducción de la mortalidad global, la incidencia general sigue inalterada en torno a 3,2 episodios por niño/año [2-5]. No obstante, es importante destacar que la cantidad de información disponible a partir de encuestas epidemiológicas fiables entre estos periodos de tiempo también varía significativamente y que, recientemente, se ha realizado un número de estudios relativamente escaso. En la encuesta previa realizada en 1993 se incluían 276 estudios efectuados en 60 países [3], mientras que la revisión de 2003 presentaba datos de solo 31 estudios realizados en 20 países [5].

Se admite que la mayoría de los trastornos diarreicos forma un continuo y que la mayoría de los casos se resuelve en el curso de la primera semana de enfermedad. No obstante, una proporción menor de procesos diarreicos no se resuelve y persiste durante más de 2 semanas. La diarrea persistente (DP) se define como episodios que, si bien se inician súbitamente, duran por lo menos 14 días [6]. Estos criterios identifican a niños con una carga diarreica considerablemente mayor y un aumento de la tasa de muertes relacionadas con diarrea. Se ha comprobado que estos episodios representan del 3 al 20\% de todos los episodios diarreicos en niños menores de 5 años [7]. En varios extensos estudios extrahospitalarios de diarrea $[8$, 9] se ha demostrado que la DP es directamente responsable del 36 al 54\% de todas las muertes relacionadas con diarrea. No obstante, se ha destacado que dichas diferencias en la mortalidad entre distintas regiones puede asociarse a características medioambientales y diferencias en la utilización de la asistencia sanitaria y el tratamiento de rehidratación oral [10]. Aunque en un extenso estudio prospectivo de diarrea realizado en el norte de India, la DP representaba solo el 5\% de todos los episodios diarreicos, la tasa de mortalidad por DP fue del $14 \%$ en comparación con el $0,7 \%$ para episodios de menor duración [11].
Es importante reconocer que la mayoría de casos de DP en países en vías de desarrollo representa diarreas postinfecciosas. La mayoría de los casos de diarrea en países en vías de desarrollo es de inicio agudo y dura menos de una semana. No obstante, un grupo de niños presenta episodios de mayor duración, algunos de los cuales persisten durante más de 14 días [12]. Aunque la división mencionada anteriormente de episodios diarreicos en agudos y DP puede ser arbitraria, esta definición operativa identifica a niños con un incremento significativo de las cargas diarreicas. En un estudio prospectivo realizado en el noreste de Brasil, Lima et al. [13] demostraron que todos los niños con una considerable carga diarreica (definida como más del 16\% de los días totales con diarrea) presentaban cuando menos un episodio de DP durante el periodo de estudio de 1 año. Observaciones similares efectuadas por Moy et al. [14] en una cohorte rural de 204 niños en Zimbabwe indicaban que, aunque la DP representaba solo el 6\% de todos los episodios diarreicos, éstos incluían el 22\% del total de días de diarrea registrado en este estudio y que estos niños también presentaban mayores tasas de recaídas de diarrea en el seguimiento. Por lo tanto, los niños con DP pueden no representar un subgrupo distinto sino formar parte de un continuo en la propensión a la diarrea. Numerosos niños (especialmente niños pequeños) en países en vías de desarrollo pueden sufrir frecuentes episodios recurrentes de diarrea aguda. Mientras que unos pocos episodios individuales pueden persistir más allá de 14 días, las recidivas frecuentes de episodios diarreicos agudos resultan en deterioro nutricional y pueden predisponer a que estos niños contraigan una DP.

En contraste con la DP en países en vías de desarrollo, casos de diarrea prolongada en niños pequeños en países desarrollados pueden corresponder a la categoría de la diarrea intratable de la lactancia. Estos casos se definen como diarreas que duran como mínimo 2 semanas y en las que puede ser necesario administrar por vía endovenosa por lo menos el 50\% de las necesidades diarias de líquidos. Muchos de estos trastornos conllevan una base genética y pueden observarse también en países en vías de desarrollo, aunque su prevalencia resulta eclipsada considerablemente por los casos de DP postinfecciosa.

\section{Métodos de revisión}

Un reciente grupo de trabajo sobre DP revisó la información y las acciones necesarias a nivel de pediatras, centros docentes y sociedades profesionales [15]. No obstan- 


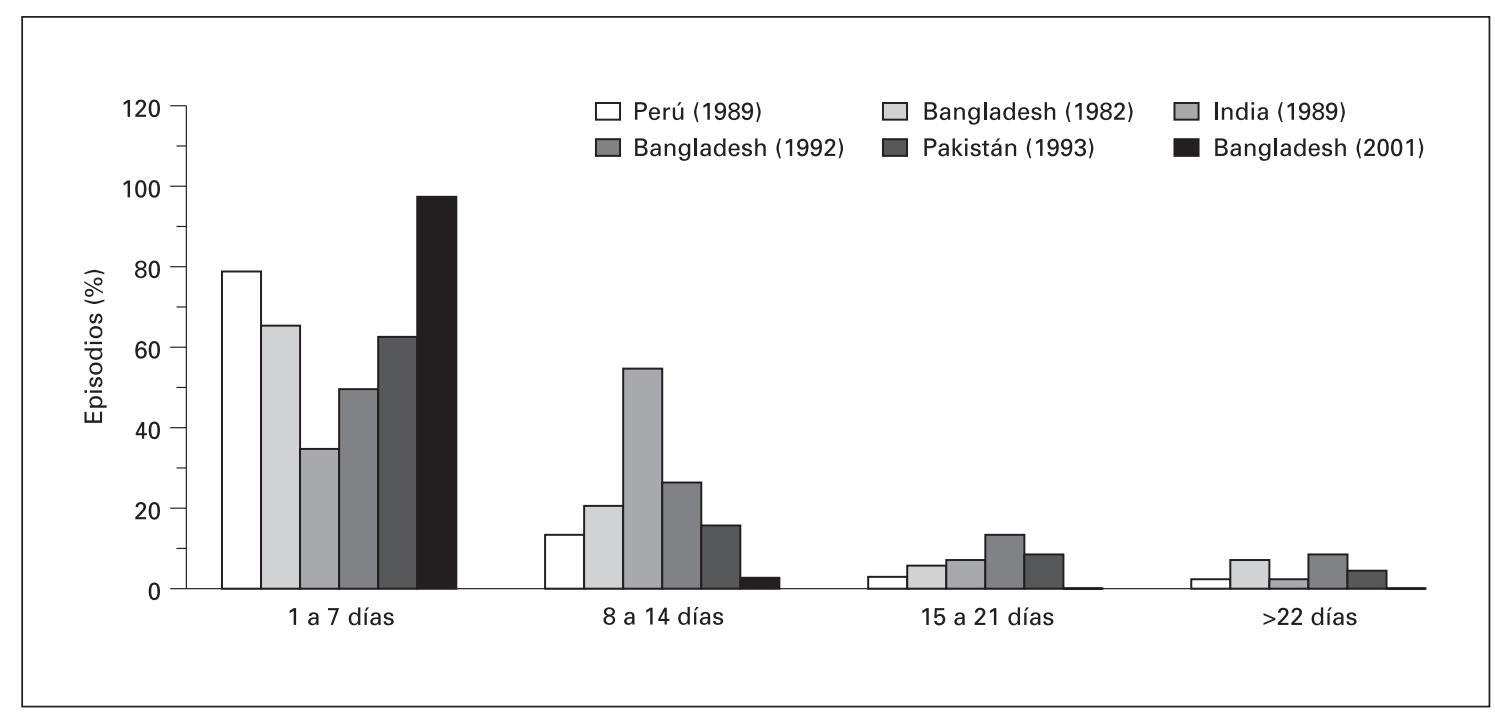

Fig. 1. Incidencia de diarrea infantil en entornos extrahospitalarios [11, 17-20].

te, la revisión no ha elaborado recientemente tendencias epidemiológicas ni intervenciones necesarias en sistemas de salud pública. Esta revisión actual presentará una perspectiva general del estado actual de la información epidemiológica y lagunas de evidencia para la asistencia clínica, así como, intervenciones de salud pública.

A fin de evaluar la información y las tendencias recientemente disponibles, revisamos toda la información existente sobre epidemiología de diarreas y DP durante los últimos 15 años (1990 a 2004) en bibliotecas electrónicas, tanto médicas (PubMed/Medline, ExtraMed, Pop Line) como de ciencias sociales. También utilizamos otros términos de búsqueda, como 'diarrea crónica', 'diarrea prolongada' y 'diarrea intratable', para proceder a la búsqueda de trabajos significativos publicados. Además de la búsqueda manual de monografías pertinentes y materiales de la OMS/UNICEF, también se procedió a acceder a la información reciente sobre la DP y estrategias de tratamiento de niños seleccionados. Seguidamente, los estudios disponibles se clasificaron en los que abordaban datos epidemiológicos o estimaciones de cargas, patogénesis y estrategias de tratamiento en centros o en entornos extrahospitalarios, así como ensayos controlados y aleatorizados disponibles. También evaluamos estudios de DP relacionados con la situación del VIH en poblaciones y patógenos relacionados como cryptosporidium, Giardia lamblia, etc ...

Resultó evidente que el grueso de la información epidemiológica sobre la relación entre episodios diarreicos agudos y DP derivaba de estudios emprendidos hace una década. Destacaba la notable escasez de datos recientes sobre esta cuestión, especialmente procedentes de zonas no endémicas de VIH. No obstante, se evidenció a partir de estudios realizados en zonas endémicas de VIH que la enteropatía crónica y la DP se identificaban crecientemente como manifestaciones comunes de infección por VIH avanzada y SIDA [16].

Se dispone en conjunto de pocas pruebas de la disminución de la incidencia de DP global real. En la figura 1 se indica la duración de la diarrea a partir de varios estudios extrahospitalarios en países en vías de desarrollo [11, 17-20]. Aunque los datos recientes de estudios extrahospitalarios de diarrea son relativamente escasos, las tasas globales de DP en entornos extrahospitalarios se manifiestan notablemente constantes durante el periodo comprendido entre 1980 y 1992. En un reciente estudio extrahospitalario en Bangladesh [20], se indica que la DP representaba menos del $0,5 \%$ de todos los casos de diarrea en un entorno extrahospitalario urbano y que es difícil generalizar sobre tendencias en la reducción de la carga de DP. Existe una necesidad evidente de revisar las proporciones estimadas previamente de episodios diarreicos agudos que han llegado a ser prolongados.

La epidemia de VIH/SIDA en África ha experimentado un cambio en los patrones etiológicos habituales de diarrea. Los episodios prolongados asociados a infecciones causadas por microorganismos relativamente raros, como cryptosporidium, se han vuelto más comunes. 
Tabla 1. Patógenos asociados a diarrea prolongada y persistente

\begin{tabular}{ll}
\hline Bacterias & Escherichia coli enteroagregativo \\
& Escherichia coli enteropatógeno \\
& Campylobacter \\
& Salmonella enteritides \\
& Especies de Shigella \\
& Clostridium difficile \\
& Arcobacter butzleri \\
& Especies de Klebsiella \\
\hline Parásitos & Giardia lamblia \\
& Blastocystis hominis ${ }^{1}$ \\
& Cryptosporidium spp. ${ }^{1}$ \\
& Entamoeba histolytica \\
& Cyclospora cayetanensis ${ }^{1}$ \\
\hline Virus & Astrovirus humano 3 \\
& Enterovirus \\
& Picobernavirus \\
\hline
\end{tabular}

${ }^{1}$ Especialmente asociado a infecciones por VIH/SIDA.

Tabla 2. Etiología de la diarrea intratable de la lactancia

1 Diarrea congénita por cloruros

2 Enfermedad de inclusión de microvellosidades

3 Displasia epitelial o enteropatía en penacho

4 Enteropatía autoinmune

5 Alergia alimentaria

6 Seudo-obstrucción intestinal crónica/enfermedad de Hirschsprung

7 Linfangiectasia intestinal

8 Abeta-lipoproteinemia

9 Enteropatía eosinofílica

10 Neurodisplasia intestinal

11 Deficiencia enzimática congénita (malabsorción congénita de glucosa y galactosa, deficiencia de sacarasa e isomaltasa, etc...)

12 Tricorrexis nudosa, dismorfismo facial, inmunodeficiencia y cirrosis

\section{Patogénesis de la DP}

La diarrea prolongada en la infancia puede derivar también de un gran número de procesos, muchos de los cuales son ajenos a las infecciones. Los más corrientes de estos trastornos son los siguientes: intolerancia a la proteína de la leche de vaca/soya (en lactantes menores de 6 meses, a menudo con deposiciones sanguinolentas), celiaquía (enteropatía sensible al gluten) y fibrosis quística (creatorrea y esteatorrea secundarias a insuficiencia pan- creática). En otros casos, también pueden observarse diarreas de niños pequeños en lactantes relativamente bien nutridos.

No obstante, en conjunto, el desencadenante subyacente más importante de la DP es un episodio diarreico agudo debido a una infección enteral y a sus consecuencias subsiguientes. Aunque se han realizado varios estudios sobre patógenos causantes de DP en países en vías de desarrollo, en pocos se ha identificado con precisión un microorganismo causal importante. Aunque pueda parecer que la DP sea una consecuencia del tratamiento deficiente de una diarrea infecciosa aguda, algunos patógenos se aíslan con mayor frecuencia en niños afectos de DP. En la tabla 1 se presentan los diversos microorganismos asociados a DP en niños de diferentes partes del mundo.

En general, casi $25 \%$ de todas las muertes por diarrea se asocia a disentería, de la que una gran proporción está causada por microorganismos Shigella. En un estudio reciente en Bangladesh [21] se diagnosticó DP en el 23\% de los niños con shigellosis, y las infecciones por Shigella fueron relacionadas significativamente con un mayor riesgo de DP (riesgo relativo ajustado por edad: 1,83 ; intervalo de confianza del 95\%: 1,19 a 2,81). Se ha observado que la infección por múltiples aislados de Shigella resistentes a antibióticos (riesgo relativo ajustado por edad: 3,76; intervalo de confianza del 95\%: 1,51 a 9,36) y la aparición de shigellosis durante la lactancia eran factores de riesgo para el inicio de una diarrea persistente por Shigella. No obstante, el $88 \%$ de los episodios de shigellosis persistente apareció en niños mayores, el 50\% se asociaba a shigellosis no disentérica y el $79 \%$ era causado por especies de Shigella distintas de Shigella dysenteriae. Otros microorganismos pueden participar en una manera similar en la inflamación de la mucosa. Un mecanismo patógeno adicional, subyacente a infecciones por Escherichia coli enteroagregativa puede comprender la inducción de IL-8 y la inflamación de la mucosa.

En contraste con la DP, se conocen mucho más sobre los mecanismos de absorción y transporte de la mucosa que subyacen a la diarrea intratable en países desarrollados. En la tabla 2 se presentan algunas de las causas principales de diarrea intratable de la lactancia.

Actualmente se admite que mutaciones del intercambiador $\mathrm{Cl}^{-} / \mathrm{HCO}_{3}^{-}$de la membrana apical ileocolónica (familia de portadores de soluto 26, gen del miembro 3 [SLC26A3, alias CLD o DRA]) resulta en la secreción neta de cloruro por el intestino y en un síndrome clínico identificado como diarrea congénita por perdida de cloruro [22]. En una manera análoga, se admite que la mal- 


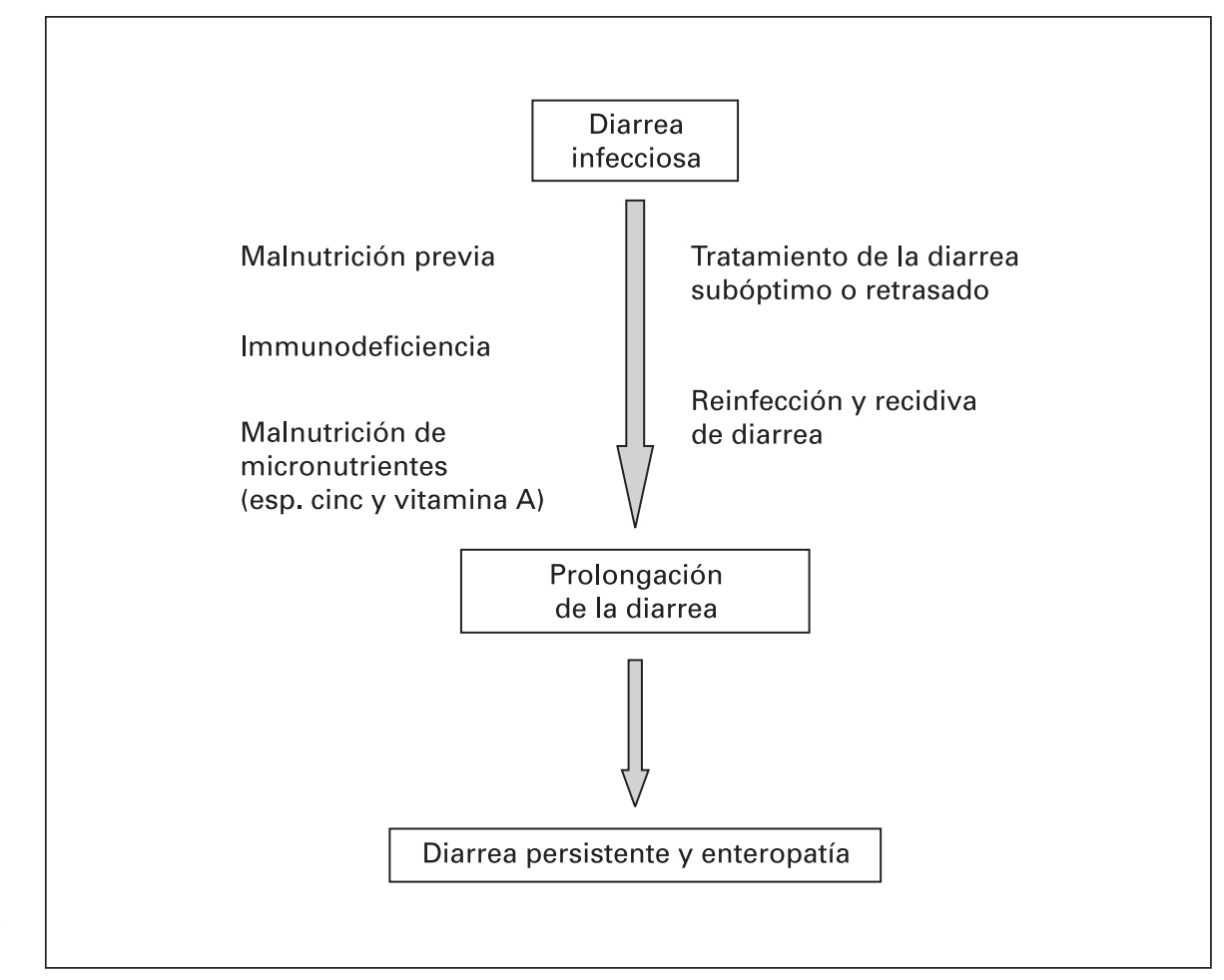

Fig. 2. Vía hacia la diarrea prolongada y persistente.

absorción congénita de glucosa/galactosa es el resultado de una anomalía genética cuya consecuencia es una reducción o la ausencia del trasportador funcional de glucosa acoplada a sodio, SGLT1, en la superficie celular del enterocito [23]; además, se ha identificado un cierto número de relaciones genotipo/fenotipo [24]. La deficiencia congénita de sacarasa e isomaltasa (DCSI) también se presenta con diarrea osmótica tras la introducción de sacarosa/almidones complejos en la dieta de destete. En estudios biológicos moleculares y celulares se ha definido con extrema precisión la patogénesis de la DCSI, demostrando que ésta parece ser una consecuencia de la síntesis defectuosa y la trasformación de glucoproteínas [25].

En contraste, los estudios detallados, realizados en países en vías de desarrollo, de anomalías mucosas y factores que subyacen a anomalías mucosas en la DP son lamentablemente raros y en ninguno se han explorado los mecanismos moleculares de la lesión intestinal prolongada. El ejemplo más llamativo del papel crítico que desempeña el sistema inmunitario en la patogénesis de la DP es la relación de VIH/SIDA con DP. Este hecho está ejemplificado por los múltiples estudios que vinculan la DP a la criptosporidiosis [26] y otras infecciones parasitarias en África y Asia. Aunque estas observaciones dan a entender que la inmunodeficiencia puede estar relacionada con el riesgo de diarrea prolongada [27], también ha suscitado un gran interés la posibilidad de que una inmunodeficiencia transitoria pueda ser asimismo un marcador de deficiencia de micronutrientes concomitante [28, 29].

Un factor adicional que predispone a la deficiente reparación intestinal puede incluir deficiencias de micronutrientes clave, que pueden influir sobre este proceso. A partir de estudios iniciales que indican el beneficio potencial del aporte complementario de cinc sobre la reducción del riesgo de diarrea prolongada [30], recientes metaanálisis del aporte complementario de cinc en enfermedades diarreicas revelan una reducción significativa de la duración y la gravedad de las enfermedades diarreicas [31]. En consecuencia, la deficiencia de cinc puede contribuir significativamente a la prolongación de la lesión de la mucosa y retrasar los mecanismos de reparación intestinal. Los beneficios del cinc en la reducción de la mortalidad por diarrea infantil han alentado a su inclusión en las recomendaciones actuales para el tratamiento de la diarrea por parte de OMS/UNICEF [32]. No obstante, se sabe muy poco acerca de los mecanismos de acción del cinc en la reducción de la duración y la gravedad de la diarrea y el mantenimiento de la integridad de la mucosa intestinal. 
La reparación intestinal deficiente se contempla como un componente clave de la morfología anormal de la mucosa. No obstante, los factores exactos que subyacen a procesos de reparación ineficaces y el mantenimiento de la lesión se conocen poco. El resultado final de la perturbación de la mucosa es la mala absorción de nutrientes luminales y el incremento de la permeabilidad del intestino para antígenos alimentarios o microbianos. Aunque la DP se identifica ampliamente como trastorno nutricional, el conocimiento de los mecanismos que predisponen a la DP y a la inmunopatología de la mucosa en niños que presentan este síndrome es notablemente precario. Sin embargo, se han identificado diversos factores que contribuyen a la persistencia de la diarrea, entre los que destacan la subnutrición general y las deficiencias de micronutrientes e inmunitarias, lo que permite suponer que el retraso de la reparación intestinal consecutivo a la lesión inicial puede ser un importante factor contributivo. En la figura 2 se ilustran los mecanismos asociados al retraso de la reparación intestinal y a la enteropatía prolongada que pueden subyacer a la DP postinfecciosa.

Un conocimiento claro de las alteraciones en la morfología y la fisiología intestinales es crucial para el desarrollo de estrategias de intervención. No obstante, en estudios bastante reducidos se ha descrito una amplia variedad de alteraciones patológicas que aparecen después de la DP [33-35]. Estos estudios presentan datos importantes indicativos de inflamación crónica y deterioro de la integridad de la mucosa. Al respecto, destaca la necesidad de un trabajo mucho más concertado en poblaciones representativas.

\section{Tratamiento y manejo de la DP}

El aspecto más importante en el tratamiento del niño con DP es una evaluación inmediata y un tamizaje para tratamiento apropriado. El tratamiento de la DP en niños subnutridos se basa en los principios del tratamiento de la diarrea y la subnutrición. Aunque un subgrupo puede estar gravemente subnutrido y requerir una rehabilitación nutricional rápida, frecuentemente en el medio hospitalario, en otros casos puede ser posible el tratamiento ambulatorio. Dado el tiempo prolongado que puede transcurrir hasta la recuperación, la hospitalización prolongada puede ser bastante problemática en países en vías de desarrollo y, siempre que sea posible, debe alentarse el tratamiento ambulatorio o domiciliario.
A continuación se presentan los principios básicos del tratamiento de la DP, y en la figura 3 se resume un enfoque terapéutico recomendado [36].

\section{(1) Reanimación inicial y estabilización}

La mayoría de los niños con DP y subnutrición asociada no está gravemente deshidratada y una rehidratación oral puede bastar. No obstante, las exacerbaciones agudas y el vómito asociado pueden exigir breves periodos de rehidratación intravenosa con lactato Ringer. Los desequilibrios graves de electrolitos, como hipopotasemia y acidosis grave, pueden requerir corrección. Cabe mencionar por su mayor importancia la identificación de translocación bacteriana [37] e infecciones sistémicas en niños con DP gravemente subnutridos, que representan una causa frecuente de mortalidad precoz. Estas circunstancias deben detectarse en el momento del ingreso. Hasta la mitad de todos los niños con DP subnutridos puede presentar infección sistémica asociada, que exige reanimación y tratamiento antimicrobiano [38, 39]. En niños subnutridos, gravemente enfermos, candidatos de hospitalización, puede indicarse la administración de antibióticos de amplio espectro en el momento del ingreso inicial y estabilización mientras se esperan los resultados de los cultivos. Algunos pueden precisar también tratamiento empírico de la criptosporidiosis, especialmente niños infectados por VIH [40].

\section{(2) Tratamiento de rehidratación oral}

Aunque, en general, la solución de rehidratación oral estándar de la OMS basta para reemplazar las pérdidas en curso, pruebas recientes indican que los líquidos de rehidratación oral de baja osmolalidad [41, 42] pueden ser ventajosos en niños subnutridos sin riesgo asociado de hiponatremia.

\section{(3) Nutrición enteral y selección de la dieta}

Es extraordinariamente raro detectar DP en lactantes alimentados exclusivamente con leche materna y, con las posibles excepciones específicas del contexto de situaciones en las que la DP acompaña a una infección por VIH adquirida perinatalmente, la lactancia materna debe continuar.

A pesar de las anomalías de la mucosa y el menoscabo de los mecanismos digestivos y absortivos, la mayoría de los niños con DP presenta una capacidad de absorción adecuada y tolera la nutrición enteral. Los estudios de balance metabólico en niños subnutridos con DP indican que con diversas dietas puede tener lugar una absorción satisfactoria de hidrato de carbono, proteína y grasa [43, 


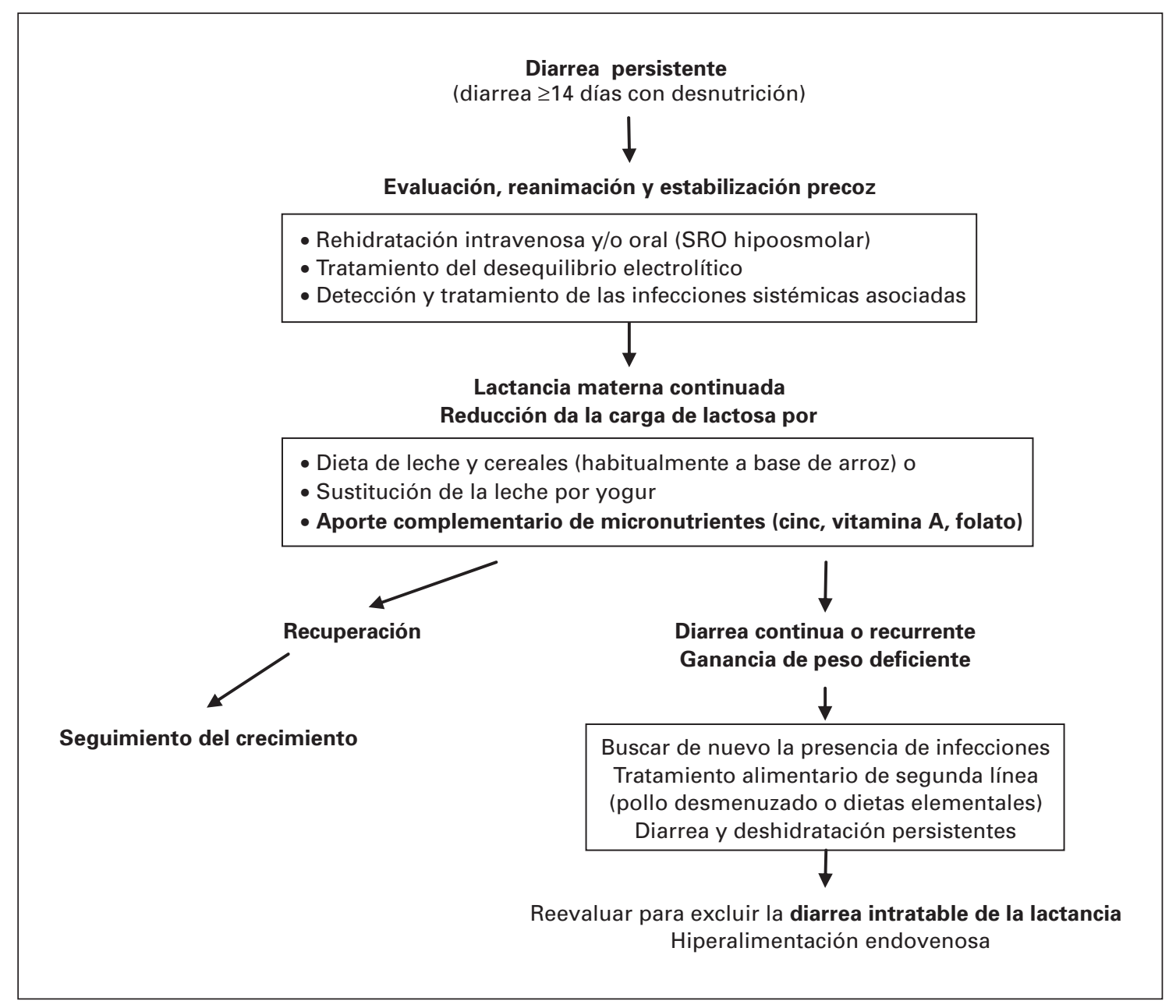

Fig. 3. Algoritmo recomendado para el tratamiento de la diarrea persistente.

44]. En consecuencia, en general la retirada de la leche y su sustitución por formulaciones especializadas (y costosas) exentas de lactosa son innecesarias.

Aunque la mayoría de los niños con DP no es intolerante a la lactosa, la administración de cargas de lactosa que superan $5 \mathrm{~g} / \mathrm{kg} /$ día se asocia a mayores tasas de purga y fracasos terapéuticos [45]. Entre las estrategias alternativas para reducir las cargas de lactosa en niños subnutridos con DP destacan la adición de leche a cereales y la sustitución de la leche por productos lácteos fermentados como el yogur. Estas intervenciones alimentarias han sido evaluadas extensamente en varios estudios, demostrando su eficacia y aceptabilidad en comparación con varias formulaciones costosas [7, 46].

En raras ocasiones, cuando la intolerancia alimentaria impide la administración de formulaciones basadas en leche de vaca o de la propia leche de vaca, puede ser necesario administrar dietas especializadas exentas de leche, como una dieta basada en pollo desmenuzado o triturado o una formulación elemental [47]. Debe puntualizarse que, aunque eficaz en algunos entornos [48], esta última es inaccesible en la mayoría de los países en vías de desarrollo. No obstante se ha comprobado que una combinación de goma guar y pollo desmenuzado es eficaz en niños con DP [49]. Además de lo indicado anteriormente, la adición de banana verde o pectina a la dieta es, según se ha demostrado, eficaz en el tratamiento de la DP [50].

La densidad calórica habitual de cualquier dieta utilizada para el tratamiento de la DP debe situarse en torno a $1 \mathrm{kcal} / \mathrm{g}$, valor encaminado a proporcionar una ingestión calórica mínima de $100 \mathrm{kcal} / \mathrm{kg} /$ día y una ingestión 
de proteína entre 2 y $3 \mathrm{~g} / \mathrm{kg} /$ día. Las formulaciones de arroz y lentejas utilizadas actualmente en el sur de Asia, como el khitchri, proporcionan esa densidad calórica en combinación con una ingestión de proteína y una proporción de aminoácidos óptimas.

\section{(4) Aporte complementario de micronutrientes}

Se admite en gran medida que la mayoría de los niños subnutridos con DP presenta deficiencias asociadas de micronutrientes, entre las que destacan las de cinc, hierro $\mathrm{y}$ vitamina A. Esto puede ser consecuencia de la ingestión deficiente y las pérdidas enterales continuadas y exige la reposición durante el tratamiento [51]. Aunque la evidencia que respalda la administración de cinc en niños con DP es convincente, cabe la probabilidad de que estos niños sufran múltiples deficiencias de micronutrientes. Se ha demostrado que la administración concomitante de vitamina A en niños con DP mejora los resultados [52, 53], especialmente en zonas endémicas de VIH [54]. En consecuencia, es importante asegurar que todos los niños con DP y subnutrición reciban una dosis inicial de 100.000 unidades de vitamina A y una ingestión diaria mínima de 3 a $5 \mathrm{mg} / \mathrm{kg} /$ día de cinc elemental.

\section{(5) Seguimiento y rehabilitación nutricional en entornos extrahospitalarios}

Si se tienen en cuenta las elevadas tasas de recaídas en la mayoría de los niños con DP, es importante abordar los factores de riesgo subyacentes e instaurar medidas preventivas. Entre éstas destacan la nutrición apropiada (lactancia materna y nutrición complementaria) y la atención minuciosa a la higiene y el saneamiento medioambientales. Esto plantea un reto considerable en comunidades carentes de necesidades básicas, como agua limpia y eliminación de aguas cloacales.

Además de los aspectos preventivos, el problema en la mayoría de los entornos es el desarrollo y el mantenimiento de una forma de tratamiento alimentario utilizando ingredientes económicos, disponibles a domicilio y culturalmente aceptables, que puedan utilizarse para tratar a niños afectos de DP. Dado que la mayoría de los casos de DP aparece en el medio extrahospitalario y que los padres son frecuentemente reticentes a recabar ayuda hospitalaria, se impone la necesidad de elaborar y poner en práctica medidas terapéuticas económicas y prácticas de carácter domiciliario. Las evidencias disponibles indican que este procedimiento puede ser completamente factible en entornos extrahospitalarios $[55,56]$.

\section{Estrategias preventivas de la DP}

Aunque la rehabilitación nutricional óptima de niños con DP se contempla como una piedra angular del tratamiento [44], la prevención es crucial. Para esta finalidad, las mejorías a gran escala de las estrategias de prevención y tratamiento de la diarrea en países en vías de desarrollo son primordiales. Tal como indicamos en la revisión de las intervenciones disponibles a favor de la supervivencia de los niños, las intervenciones disponibles pueden prevenir casi el 55\% de todos los fallecimientos globales en niños menores de 5 años [57], 20\% de los cuales está relacionado con la diarrea.

Entre estas intervenciones destacan la lactancia materna exclusiva y estrategias de nutrición complementaria seguras para prevenir la diarrea y, en caso de que aparezcan episodios diarreicos, un tratamiento óptimo con mejoría de la rehidratación oral, aporte suplementario de cinc y nutrición continuada. Tal como se ha indicado anteriormente, esto exige que todos los episodios de diarrea se identifiquen con prontitud y se traten óptimamente con rehidratación oral y nutrición enteral mediante dietas económicas, disponibles a domicilio y culturalmente aceptables, que puedan utilizarse para tratar a niños con DP en entornos ambulatorios. Las etapas siguientes consistirían en integrar estas intervenciones, de manera que las familias y los asistentes sanitarios extrahospitalarios pudieran ponerlas en práctica en diversas situaciones [58].

La provisión de intervenciones preventivas y terapéuticas esenciales para reducir la DP en los sistemas sanitarios es necesaria a fin de alcanzar las metas de desarrollo del milenio de reducir la mortalidad infantil en $2 / 3$ en el año 2015. La introducción prematura y poco higiénica de leches distintas de la leche materna y los episodios de diarrea aguda recurrente, tratados de un modo deficiente, son importantes factores predisponentes para el desarrollo de la DP. Es importante que estos factores sean evitados. Dichos factores de riesgo son generalmente prevalentes en comunidades pobres y, tanto el alivio de la pobreza como los mecanismos de apoyo al sector social, revisten una importancia primordial. Por lo tanto, la promoción de la lactancia materna exclusiva durante un mínimo de 6 meses, la evitación de una nutrición con formulaciones antihigiénicas y el destete oportuno y adecuado con alimentos nutritivos e higiénicos contribuirán a prevenir los episodios de DP postinfecciosa. Esto debe asociarse a medidas de control medioambiental y al abastecimiento de agua y comida fiables [59]. En ausencia de estas últimas, las intervenciones para fomentar las estra- 
tegias de lavado de las manos y medidas domesticas para purificar el agua son claves para reducir la carga de diarrea $[60,61]$.

Una combinación de asignación de recursos apropiados para la salud pública y necesidades básicas, formación del personal y movilización de la comunidad es necesaria para reducir la carga global de diarrea y DP. Esto requiere esfuerzos persistentes y concertados. En numerosas partes del mundo en vías de desarrollo, estas intervenciones exigen que, aparte de las intervenciones biomédicas, concedamos una importancia primordial al alivio de la pobreza y al fomento de un programa de derechos humanos y equidad.

\section{Bibliografía}

1 Black RE, Morris SS, Bryce J: Where and why are 10 million children dying every year? Lancet 2003;361:2226-2234.

2 Snyder JD, Merson MH: The magnitude of the global problem of acute diarrheal disease: a review of active surveillance data. Bull World Heath Organ 1982;60:605-613.

- 3 Bern C, Martines J, de Zoysa I, Glass RI: The magnitude of the global problem of diarrhoeal disease: a ten-year update. Bull World Health Organ 1992;70:705-714.

4 Yusufzai M, Bhutta ZA: Global burden of childhood diarrhea; in Bhutta ZA (ed): Contemporary Issues in Childhood Diarrhea and Malnutrition. Karachi, Oxford University Press, 2000, pp 1-22.

5 Kosek M, Bern C, Guerrant RL: The global burden of diarrheal disease as estimated from studies published between 1990 and 2000. Bull World Health Organ 2003;81:197-2004.

-6 Anonymous: Persistent diarrhoea in children in developing countries: memorandum from a WHO Meeting. Bull World Health Organ 1988;66:709-717.

7 Anonymous: Evaluation of an algorithm for the treatment of persistent diarrhoea: a multicentre study. International Working Group on Persistent Diarrhoea. Bull World Health Organ 1996;74:479-489.

$\checkmark 8$ Schorling JB, Wanke CA, Schorlling SK, McAullife JF, de Souza MA, Guerrat RL: A prospective study of persistent diarrhea among children in an urban Brazilian slum: patterns of occurrence and etiologic agents. Am J Epidemiol 1990;132:144-156.

-9 Fauveau V, Henry FJ, Briend A, Yunus M, Chakraborty J: Persistent diarrhea as a cause of childhood mortality in rural Bangladesh. Acta Paediatr Suppl 1992;381:12-14.

10 Victora CG, Huttly SRA, Fuch SC, et al: International differences in clinical patterns of diarrhoeal deaths: a comparison of children from Brazil, Senegal, Bangladesh and India. J Diarrhoeal Dis Res 1993;11:25-29.

-11 Bhan MK, Bhandari N, Sazawal S, Clemens J, Raj P: Descriptive epidemiology of persistent diarrhoea among young children in rural northern India. Bull World Health Organ 1989;67:281-288.

12 Black RE: Persistent diarrhea in children of developing countries. Pediatr Infect Dis J 1993; 12:751-761.
13 Lima AAM, Fang G, Schorling JB, Albuquerque L, Mc Aulliffe JA, Mota S, Leite R, Guerrant RL: Persistent diarrhea in northeast Brazil: etiologiesandinteraction with malnutrition. Acta Paediatr Suppl 1992;381:39-44.

14 Moy RJ, Booth IW, Choto R-G, McNeish AS: Recurrent and persistent diarrhoea in a rural Zimbabwean community: a prospective study. J Trop Pediatr 1991;37:293-299.

15 Bhutta ZA, Ghishan F, Lindley K, Memon IA Mittal S, Rhoads JM: Persistent and chronic diarrhea and malabsorption: Working Group report of the second World Congress of Pediatric Gastroenterology, Hepatology, and $\mathrm{Nu}-$ trition. J Pediatr Gastroenterol Nutr 2004; (suppl 2):S711-S716.

16 Adejuyigbe EA, Oyelami O, Onayemi O, Durosinmi MA: Paediatric HIV/AIDS in Ile-Ife, Nigeria. Cent Afr J Med 2003;49:74-78.

17 Black RE, Brown KH, Becker S, Yunus M: Longitudinal studies of infectious diseases and physical growth of children in rural Bangladesh. I. Patterns of morbidity. Am J Epidemiol 1982;115:305-314.

18 Lanata CF, Black RE, Gilman RH, Lazo F, Del Aguila R: Epidemiologic, clinical, and laboratory characteristics of acute vs persistent diarrhea in periurban Lima, Peru. J Pediatr Gastroenterol Nutr 1991;12:82-88.

19 Mahmud A, Jalil F, Karlberg J, Lindblad BS: Early child health in Lahore, Pakistan: VII. Diarrhoea. Acta Paediatr Suppl 1993;390:7985.

20 Haque R, Monda Dl, Kirkpatrick BD, Akther Farr BM, Sack RB, Petri WB Jr: Epidemiologic and clinical characteristics of acute diarrhea with emphasis on Entamoeba histolytica infections in preschool children in an urban slum of Dhaka, Bangladesh. Am J Trop Med Hyg 2003;69:398-405.

21 Ahmed F, Ansaruzzaman M, Haque E, Rao MR, Clemens JD: Epidemiology of postshigellosis persistent diarrhea in young children. Pediatr Infect Dis J 2001;20:525-530.

22 Hoglund P, Haila S, Socha J, Tomaszewski L, Saarialho-Kere U, Karjalainen-Lindsberg ML, Airola K, Holmberg C, de la CA, Kere J: Mutations of the Down-regulated in adenoma (DRA) gene cause congenital chloride diarrhoea. Nat Genet 1996;14:316-319.
23 Turk E, Zabel B, Mundlos S, Dyer J, Wright EM: Glucose/galactose malabsorption caused by a defect in the $\mathrm{Na}+$ /glucose cotransporter. Nature 1991;350:354-356.

-24 Martin MG, Turk E, Lostao MP, Kerner C, Wright EM: Defects in Na+/glucose cotransporter (SGLT1) trafficking and function cause glucose-galactose malabsorption. Nat Genet 1996; 12:216-220.

25 Naim HY, Roth J, Sterchi EE, Lentze M, Milla P, Schmitz J, Hauri HP: Sucrase-isomaltase deficiency in humans. Different mutations disrupt intracellular transport, processing, and function of an intestinal brush border enzyme. J Clin Invest 1988;82:667-679.

26 Amadi B, Kelly P, Mwiya M, Mulwazi E, Sianongo $\mathrm{S}$, Changwe $\mathrm{F}$, Thomson $\mathrm{M}$, Hachungula J, Watuka A, Walker-Smith J, Chintu C: Intestinal and systemic infection, HIV, and mortality in Zambian children with persistent diarrhea and malnutrition. J Pediatr Gastroenterol Nutr 2001;32:550-554.

-27 Baqui AH, Black R E, Sack R B, Chowdhury HR, Yunus M, Siddique AK: Malnutrition, cell-mediated immune deficiency and diarrhea: a community-based longitudinal study in rural Bangladeshi children. Am J Epidemiol 1993; 137:355-365.

-28 Azim T, Ahmad SM, Sefat-E-Khuda, Sarker MS, Unicomb LE, De S, Hamadani JD, Salam MA, Wahed MA, Albert MJ: Immune response of children who develop persistent diarrhea following rotavirus infection. Clin Diagn Lab Immunol 1999;6:690-695.

29 Taniguchi K, Rikimaru T, Yartey JE, Akpedonu P, Armar-Klemesu MA, Nkrumah FK, Kamiya H, Kishi K, Armar DA: Immunological background in children with persistent diarrhea in Ghana. Pediatr Int 1999;4:162167.

30 Sazawal S, Black R E, Bhan M K, Bhandari N, Sinha A, Jalla S: Zinc supplementation in young children with acute diarrhea in India. N Engl J Med 1995;333:839-844.

31 Bhutta ZA, Bird SM, Black RE, Brown KH, Gardner JM, Hidayat A, Khatun F, Martorell R, Ninh NX, Penny ME, Rosado JL, Roy SK, Ruel M, Sazawal S, Shankar A: Therapeutic effects of oral zinc in acute and persistent diarrhea in children in developing countries: pooled analysis of randomized controlled trials. Am J Clin Nutr 2000;72:1516-1522. 
32 WHO/UNICEF Joint Statement: Clinical Management of Acute Diarrhea. May 2004. http://www.mostproject.org/ZINC/WHOUNICEF/Statement.pdf (accessed Feb 27, 2005).

33 Sullivan PB: Studies of the small intestine in persistent diarrhea and malnutrition: the Gambian experience. J Pediatr Gastroenterol Nutr 2002;34(suppl 1):S11-S13.

-34 Campbell DI, Murch SH, Elia M, Sullivan PB, Sanyang MS, Jobarteh B, Lunn PG: Chronic T cell-mediated enteropathy in rural west African children: relationship with nutritional status and small bowel function. Pediatr Res 2003;54:306-311.

35 Fagundes-Neto U, De Martini-Costa S, Pedroso MZ, Scaletsky IC: Studies of the small bowel surface by scanning electron microscopy in infants with persistent diarrhea. Braz J Med Biol Res 2000;33:1437-1442.

36 Bhutta ZA: Persistent diarrhea; in Guandalini S (ed): Text Book of Gastroenterology. London, Taylor \& Francis, 2004.

37 Campbell DI, Elia M, Lunn PG: Growth faltering in rural Gambian infants is associated with impaired small intestinal barrier function, leading to endotoxemia and systemic inflammation. J Nutr 2003;133:1332-1338.

- 38 Bhutta ZA, Nizami SQ, Thobani S: Factors determining recovery during nutritional therapy of persistent diarrhoea: the impact of diarrhoea severity and intercurrent infections. Acta Paediatr 1997;86:796-802.

39 Alam NH, Faruque AS, Dewan N, Sarker SA Fuchs GJ: Characteristics of children hospitalized with severe dehydration and persistent diarrhoea in Bangladesh. J Health Popul Nutr 2001;19:18-24.

40 Amadi B, Mwiya M, Musuku J, Watuka A Sianongo S, Ayoub A, Kelly P: Effect of nitazoxanide on morbidity and mortality in Zambian children with cryptosporidiosis: a randomised controlled trial. Lancet 2002;360: 1375-1380.

-41 Sarker SA, Mahalanabis D, Alam NH, Sharmin S, Khan AM, Fuchs GJ: Reduced osmolarity oral rehydration solution for persistent diarrhea in infants: a randomized controlled clinical trial. J Pediatr 2001;138:532-538.
42 Dutta P, Mitra U, Dutta S, Manna B, Chatterjee MK, De A, Bhattacharya SK: Hypo-osmolar oral rehydration salts solution in dehydrating persistent diarrhoea in children double-blind, randomized, controlled clinical trial. Acta Paediatr 2000;89:411-416.

43 Bhutta ZA, Molla AM, Isani Z, Badruddin S, Hendricks K, Snyder JD: Dietary management of persistent diarrhea: comparison of a traditional rice-lentil based diet with soy formula. Pediatrics 1991;88:1010-1018.

-44 Bhutta ZA, Hendricks KH: Nutritional management of persistent diarrhea in childhood: a perspective from the developing world. J Pediatr Gastroenterol Nutr 1996;22:17-37.

45 Penny ME, Paredes P, Brown KH: Clinical and nutritional consequences of lactose feeding during persistent postenteritis diarrhea. Pediatrics 1989;84:835-844.

46 Ashraf H, Ahmed S, Fuchs GJ, Mahalanabis D: Persistent diarrhoea: associated infection and response to a low lactose diet. J Trop Pediatr 2002;48:142-148

47 Godard C, Bustos M, Munoz M, Nussle D: Value of a chicken-based formula for refeeding of children with protracted diarrhea and malnutrition in a developing country. J Pediatr Gastroenterol Nutr 1989;9:473-480.

48 Amadi B: Role of food antigen elimination in treating children with persistent diarrhea and malnutrition in Zambia. J Pediatr Gastroenterol Nutr 2002;34(suppl 1):S54-S56.

49 Alam NH, Meier R, Sarker SA, Bardhan PK, Schneider H, Gyr N: Partially hydrolysed guar gum supplemented comminuted chicken diet in persistent diarrhoea: a randomised controlled trial. Arch Dis Child 2005;90:195199.

50 Rabbani GH, Teka T, Zaman B, Majid N, Khatun M, Fuchs GJ: Clinical studies in persistent diarrhea: dietary management with green banana or pectin in Bangladeshi children. Gastroenterology 2001;121:554-560.

51 Mahalanabis D, Bhan MK: Micronutrients as adjunct therapy of acute illness in children: impact on the episode outcome and policy implications of current findings. Br J Nutr 2001; 85(suppl 2):S151-S158.
52 Rahman MM, Vermund SH, Wahed MA, Fuchs GJ, Baqui AH, Alvarez JO: Simultaneous zinc and vitamin A supplementation in Bangladeshi children: randomised double blind controlled trial. BMJ 2001;323:314 318

53 Khatun UH, Malek MA, Black RE, Sarkar NR, Wahed MA, Fuchs G, Roy SK: A randomized controlled clinical trial of zinc, vitamin A or both in undernourished children with persistent diarrhea in Bangladesh. Acta Paediatr 2001;90:376-380.

54 Villamor E, Mbise R, Spiegelman D, Hertzmark E, Fataki M, Peterson KE, Ndossi G, Fawzi WW: Vitamin A supplements ameliorate the adverse effect of HIV-1, malaria, and diarrheal infections on child growth. Pediatrics 2002:109:E6

55 Bhandari N, Bahl R, Saxena M, Taneja S, Bhan MK: Prognostic factors for persistent diarrhoea managed in a community setting. Indian J Pediatr 2000;67:739-745.

- 56 Valentiner-Branth P, Steinsland H, Santos G, Perch M, Begtrup K, Bhan MK, Dias F, Aaby P, Sommerfelt H, Molbak K: Communitybased controlled trial of dietary management of children with persistent diarrhea: sustained beneficial effect on ponderal and linear growth. Am J Clin Nutr 2001;73:968-974.

57 Jones G, R Steketee RW, Black RE, Bhutta ZA, Morris SS and the Bellagio Child Survival Study Group: How many child deaths can we prevent this year? Lancet 2003;362:65-71.

58 Travis P, Bennett S, Haines A, Pang T, Bhutta Z A, Hyder AA, Pielemeier NR, Mills A, Evans T: Overcoming health-systems constraints to achieve the Millennium Development Goals. Lancet 2004;364:900-906.

59 Sheth M, Obrah M: Diarrhea prevention through food safety education. Indian J Pediatr 2004; 71:879-882.

60 Curtis V, Cairncross S: Effect of washing hands with soap on diarrhoea risk in the community: a systematic review. Lancet Infect Dis 2003;3: 275-281

61 Luby SP, Agboatwalla M, Painter J, Altaf A, Billhimer WL, Hoekstra RM: Effect of intensive handwashing promotion on childhood diarrhea in high-risk communities in Pakistan: a randomized controlled trial. JAMA 2004;291: 2547-2554. 